\title{
Gabapentin enacarbil extended release for the treatment of postherpetic neuralgia in adults
}

This article was published in the following Dove Press journal:

Therapeutics and Clinical Risk Management

30 November 2013

Number of times this article has been viewed

\section{Ben MThomas' \\ Paul Farquhar-Smith ${ }^{2}$ \\ 'Chelsea Westminster Hospital, London, UK; ${ }^{2}$ Department of Anaesthetics, The Royal Marsden NHS Foundation Trust, London, UK}

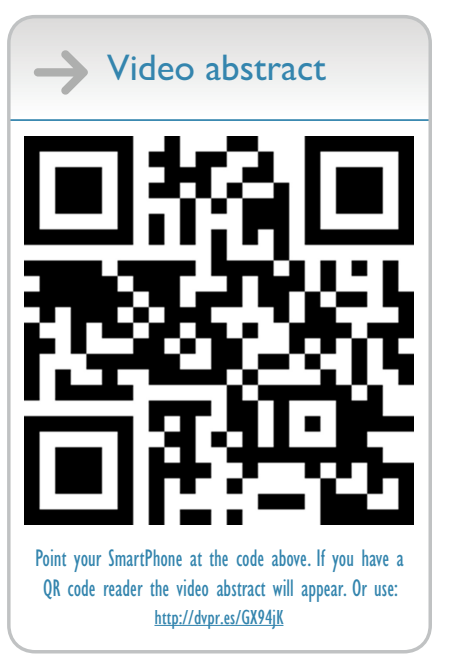

Correspondence: Paul Farquhar-Smith Department of Anaesthetics, The Royal Marsden NHS Foundation Trust, Fulham Road, London, SW3 6JJ, UK Tel +44 207352 8I7I ext 2727 Fax +44 2078082726

Email paul.farquhar-smith@rmh.nhs.uk

\begin{abstract}
The development of biomedical technology is allowing refinement of drug therapies in order to improve medication profiles and benefit patients. Gabapentin (Gp) is a medication licensed globally for various indications, including postherpetic neuralgia. It has a pharmacokinetic profile which has been suggested may limit its clinical effects and reduce medication compliance. In 2012, the US Food and Drug Administration licensed a novel preparation which aims to circumvent these limitations. Gp enacarbil is a prodrug of $\mathrm{Gp}$, which is additionally prepared in an extended release preparation. The resulting compound has an improved absorption profile and a reduced dosing frequency in comparison to immediate release Gp. An absence of comparative data, however, limits the direct evaluation of the medication to both immediate release and other extended release preparations available on the market. Additionally, no data are currently available addressing efficacy, tolerability, or side effects with other first line treatments of postherpetic neuralgia. Additional experimental data should be sought to clarify the position of $\mathrm{Gp}$ enacarbil, both within postherpetic neuralgia treatment protocols and in relation to the increasing numbers of gabapentinoids available.
\end{abstract}

Keywords: postherpetic neuralgia, garbapentin enacarbil, herpes zoster

\section{Introduction}

Acute herpes zoster (HZ) infection carries a risk of persistent and distressing chronic pain development, with $10 \%-20 \%$ of sufferers developing the symptoms of postherpetic neuralgia (PHN). ${ }^{1}$ Vigilance and early treatment of the acute infection can result in a reduced incidence of the chronic symptoms of PHN ${ }^{2}$ The majority of PHN sufferers are aged over 50 years, with the incidence doubling by the age of 80 years. ${ }^{3}$ Inflammation of the entire nerve results from the reactivation and replication of the varicella zoster virus, which in many cases had infected the host years earlier, and causes the acute pain and characteristic dermatomal rash. ${ }^{4}$

PHN is characterized by pain or dysesthesia remaining 12 weeks after rash resolution, although there are several variants of the definition. The mean length of a herpes zoster infection in the United Kingdom is 2 weeks, while the average duration of symptoms in the event of PHN development is 1.4 years. ${ }^{5}$ Licensed PHN treatments encompass various administrative routes and drug types. The pharmacokinetic profile of the immediate release form of gabapentin (GpIR) requires that its administration be in divided daily doses, with poor medication compliance being suggested as a result. ${ }^{6}$ Novel approaches to reduce dosing regimens have led to development of mechanisms to provide extended release preparations, and gabapentin (Gp) is now 
marketed in various forms. The licensing of Gp (HORIZANT $^{\circledR}$; GlaxoSmithKline, Brentford, UK) by the US Food and Drug Administration (FDA) in June 2012 extended the license from treatment for restless leg syndrome (RLS) to treatment of PHN. It provided another alternative to the immediate release (IR) preparation of Gp (Neurontin ${ }^{\circledR}$; Pfizer, New York, NY, USA), and the extended release Gp preparations (GpGr) (GRALISE ${ }^{\circledR}$; Depomed Inc, Newark, CA, USA).

\section{The management of PHN in adults}

Strategies to manage PHN take three approaches; the first is aimed at prevention of the $\mathrm{HZ}$ infection, the second targets prompt and early treatment of acute $\mathrm{HZ}$ reactivation, and the third is management of the condition itself on occurrence. Primary prevention aims to reduce the incidence of the initial infection, thus avoiding PHN development, while secondary prevention uses early treatment in order to achieve a reduction in neuropathic complications at a later stage. Approaches have included $\mathrm{HZ}$ vaccination, antiviral agents, and tricyclic antidepressant (TCA) medication. There is no consensus for worldwide treatment guidelines specifically for PHN, although various bodies provide guidance for the general treatment of neuropathic pain. In 2007, an international, evidence-based guideline recommended that first line therapies for neuropathic pain should be antidepressants (TCA or selective serotonin and noradrenaline reuptake inhibitors), gabapentinoids, or topical lidocaine. ${ }^{7}$ The revised guidelines in 2010 came to the same conclusions. ${ }^{8}$ The American Society of Anesthesiologists Chronic Pain task force suggests first line therapy to be single or multiple agents including a secondary amine TCA (nortriptyline, desipramine, or amitriptyline), a selective serotonin and noradrenaline reuptake inhibitor (duloxetine, venlafaxine), or the gabapentinoids (Gp or pregabalin). ${ }^{9}$ There are few data on how closely these guidelines are followed, yet it is clear that the actual treatments given are influenced by local factors such as availability of medicines, costs and funding, and population characteristics.

The European Federation of Neurological Societies recommend a TCA, Gp, or pregabalin as first line therapy for the diagnosis of PHN, and they mention extended release preparations but do not specifically name any. ${ }^{10}$ To date, no licensing of extended release preparations has occurred in Europe. The GpIR, Neurontin ${ }^{\circledR}$, has been a US FDA-approved treatment for PHN since 2002; an extended release preparation of Gp (GRALISE ${ }^{\circledR}$, GpGr) has been licensed since 2011. ${ }^{11}$

\section{The pharmacology, mode of action, and pharmacokinetics of gabapentin enacarbil extended release}

Gp is structurally similar to gamma-aminobutyric acid (GABA) but does not bind to GABA-A or GABA-B receptors. Multiple mechanisms of action have been postulated, including combined increased GABA synthesis, non-Nmethyl-D-aspartate receptor antagonism, and an inhibitory action at the voltage gated calcium channel ( $\alpha_{2} \delta$ subunit). Action of Gp has been demonstrated at spinal, supraspinal, and peripheral sites. Calcium channel binding has several effects, including inhibition of the release of excitatory neurotransmitters, modulation of GABAergic, glutaminergic, and monoamine function, and it may also antagonize synaptogenesis. ${ }^{12,13}$ Absorption is via a low capacity, potentially saturable mechanism, which occurs in a proximal site, specifically the duodenum and jejunum. In combination with the probable active L-amino acid transport mechanism, there is a resulting dose-dependent absorption which occurs in a narrow window of luminal transit. Bioavailability therefore decreases in the face of increasing dose, from $60 \%$ at $300 \mathrm{mg}$ to $35 \%$ or less at higher doses. It has been postulated that the resulting interpatient variability may result in suboptimal exposure to the drug in some recipients. ${ }^{14}$ The combination of a dose-dependent bioavailability and an in vivo half-life of 5-7 hours results in the administration of GpIR being recommended three or four times daily. ${ }^{15} \mathrm{Gp}$ is not metabolized in humans and depends upon renal function for excretion.

As pharmacological technology has evolved, new mechanisms for adapting pharmacokinetics have aimed to bypass factors that have previously placed limitations on drug profiles and may result in improvement upon the original drug. The Gp enacarbil (GpEn) formulation (developed under XP13512/GSK1838262; GlaxoKlineSmith, Brentford, UK) aims to do this by overcoming absorption limitations. It is an (acyloxy)alkyl carbamate prodrug of $\mathrm{Gp}$, with an absorption profile stated to provide predictable bioavailability and sustained, dose-proportional drug exposure. ${ }^{16}$ Unlike GpIR, it is absorbed via mechanisms present throughout the intestine. ${ }^{14}$ This plentiful absorptive target area makes the drug amenable to an extended release approach, and the GpEn preparation utilizes an erosion-based, extended release formula based on a wax matrix. ${ }^{16}$ Dosing of the medication is suggested at $600 \mathrm{mg}$ twice daily (bd) for PHN, initiated at $600 \mathrm{mg}$ once daily (in the morning) for 3 days, and then increased to bd $(1,200 \mathrm{mg})$ on the fourth day. ${ }^{17}$

Twelve Phase I-III trials were conducted between healthy volunteers and a population with RLS, with pharmacokinetic 
data being modeled from the findings using nonlinear, mixed effect modeling. A total of 1,341 concentration values of Gp from 95 subjects in Phase I studies and 6,873 plasma samples from 899 subjects in Phase II/III studies were included in the population analysis. Pharmacodynamic data were evaluated directly. ${ }^{12}$ The studies were examined over extended release GpEn doses of 300-2,400 mg daily.

At physiological $\mathrm{pH}$ ranges and temperature, the prodrug was identified as stable, with rapid metabolism to Gp and stable inactive byproducts. Gp production was via hydrolysis, which occurred rapidly in a number of tissue types postabsorption, most notably by nonspecific carboxylesterases in intestinal enterocytes. Absorption was by both passive and active mechanisms via high-capacity nutrient transporters, monocarboxylate transporter-1 and sodium-dependent multivitamin transporters. These transporters are known to be present throughout the upper and lower intestinal tract. ${ }^{16}$ Absorption and hydrolysis of the prodrug lead to Gp plasma concentrations increasing and declining in an exponential manner. Mean absorption lag time was 0.4 hours, with absorption maintained over 7 hours with $22 \%$ variability between subjects. Dose proportionality was present in single and multiple dose models across the tested dose range. The half-life of Gp resulting from GpEn administration is approximately 6 hours, similar to that of GpIR. Volume of distribution is $76 \mathrm{~L}$, with less than $3 \%$ protein binding. ${ }^{18}$ Neither GpEn nor Gp are substrate, inhibitor, or inducer of the major cytochrome P450 enzymes. Elimination half-life of GpEn was 6 hours, similar to GpIR. No age-, weight-, or sex-related dosing adjustments are advised. Excretion of GpEn-produced Gp is mainly via renal clearance (94\%), with elimination proportional to creatinine clearance. ${ }^{19}$ Renal excretion is primarily in the form of unchanged drug, without any further metabolism. A further $5 \%$ is elimination in the feces. ${ }^{18}$ In vivo investigation suggested dosing should be adjusted in renal failure, dependent upon calculated creatinine clearance of the individual. GpEn is licensed for both PHN and RLS, and different dosing scales exist according to the indication for prescription. ${ }^{17}$ The combined findings of the similarities of Gp provided as GpEn with the extended absorption site and the subsequent formulation into extended release form, make the basis of the reduced dosing frequencies.

\section{Efficacy studies}

The hypothesized benefits of the GpEn formulation include a reduced interpatient variability of absorption and a resultant improvement in efficacy, as suboptimal drug exposure is reduced. Additionally, by producing an extended release

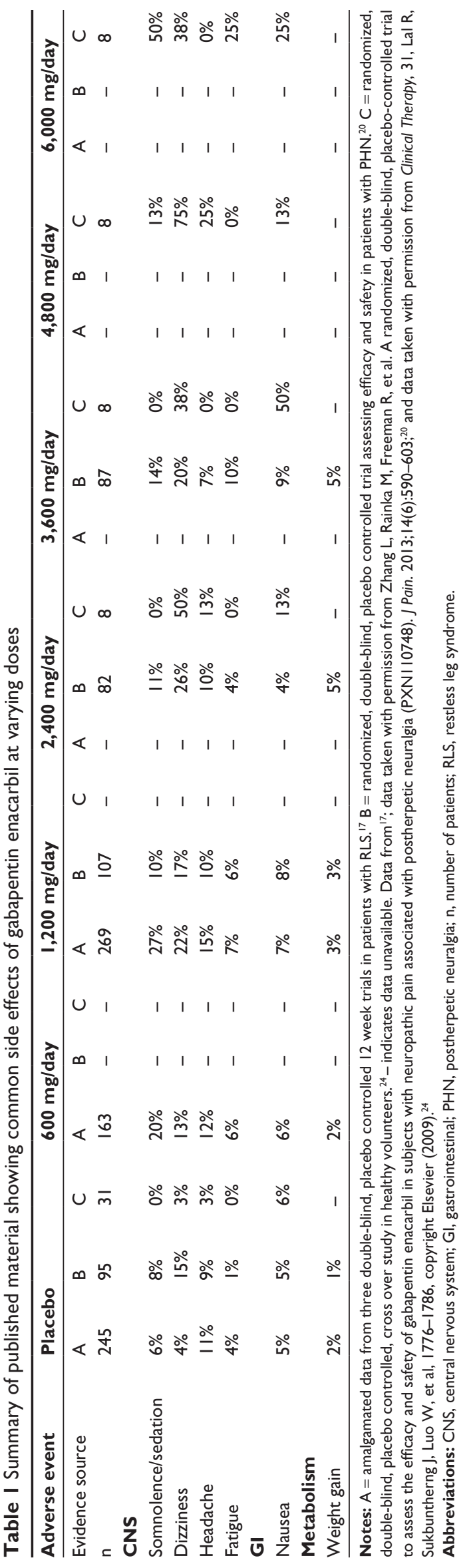


preparation, the potential for furthering this effect offers the possibility of reduced dosing frequencies.

Primarily, GpEn has been investigated in the treatment of RLS, with less evidence for its use in PHN. US FDA approval was given for PHN based on a single multicenter, randomized, double-blind, parallel group, placebo-controlled study. This study evaluated efficacy, safety, and dose response over 12 weeks. ${ }^{20} \mathrm{~A}$ second trial was also identified comparing GpEn with placebo, although the study period was much shorter at 11 days. The US FDA has also approved the use of GpEn for RLS.

Zhang et al administered doses of 1,200 mg, 2,400 mg, and 3,600 mg to 107,82 , and 87 patients with $\mathrm{PHN}$, respectively; an additional 95 patients received placebo. ${ }^{20}$ The subjects ranged in age from 18-92 years. The populations were required to have a minimum baseline 24-hour average pain intensity numerical rating scale of at least 4 . The 12-week study had an additional 1-week up-titration period and a subsequent 1-week down-titration period. The primary efficacy outcome was change from baseline in mean 24-hour average pain intensity score at the end of maintenance treatment. The results for all 371 subjects showed a statistically significant mean (standard error) reduction of -2.47 (0.204) in the $1,200 \mathrm{mg}$ group, $-2.36(0.237)$ for the $2,400 \mathrm{mg}$ group, and -2.77 (0.227) for the $3,600 \mathrm{mg}$ group compared to -1.66 (0.216) in the placebo group. A benefit was observed from as early as week 1 . Secondary outcomes were improvement in 24-hour average pain intensity scores, and a 30\% improvement was identified in $54 \%$ of the $1,200 \mathrm{mg}$-treated group, $59 \%$ in the $2,400 \mathrm{mg}$ group, and $60 \%$ in the $3,600 \mathrm{mg}$ group, compared to $42 \%$ for the placebo-treated group. A further $35 \%-43 \%$ of patients across all groups reported at least a $50 \%$ reduction compared to $23 \%$ in the placebo group. Comment was made that the study was not powered to compare between groups and that no benefit was demonstrated between dosing groups. ${ }^{20}$ While more patients in these treatment groups achieved a sustained improvement in these secondary outcome measures, it was not stated that the results achieved statistical significance. Rescue medication (paracetamol up to $3 \mathrm{~g}$ daily) use was lower across all three treatment groups than for placebo, but again showed no statistical significance. No significant differences in electrocardiogram, vital signs, and laboratory assessments were noted between groups. Several multiple serious adverse events were reported but gastritis was the only serious adverse event clearly attributable to the treatment. The lowest incidence of trial withdrawal was in the $1,200 \mathrm{mg}$ group (6\%) compared to $18 \%$ for the $3,600 \mathrm{mg}$ group and $13 \%$ for the placebo-treated group. The study concluded that twice-daily dosing of GpEn was efficacious in relieving pain and improving sleep in its study group, which was considered representative of the patient population with $\mathrm{PHN}$.

The second GpEn versus placebo trial published was by Backonja et al, and it was also a double-blind, randomized trial, but with methodological differences compared to the first study. ${ }^{21}$ In contrast to the Zhang el al ${ }^{20}$ paper (where a washout period of between 14 days and 28 days was required, depending on medication type) this study enforced a weeklong washout period, followed by the establishment of the patients on open-label GpIR for 11 days before undertaking a 14-day trial of GpEn (1,200 mg) against placebo. In this study, 101 patients were randomized; 47 received GpEn and 54 received placebo. The subjects treated with GpEn ranged in age from 33-82 years, and had a significantly greater decrease in mean \pm standard deviation weekly pain scores compared with the placebo-treated group; $-2.1 \pm 1.63$ versus $-1.2 \pm 1.69(P=0.0321)$. In addition, following the same mean change from baseline during the open label period, patients randomized to the study drug were observed to have a significant improvement in outcome compared to their period on $\mathrm{Gp}$. The trial also evaluated self-reported pain, sleep, mood, global improvement, and adverse events compared against placebo. ${ }^{21}$ Significant improvements from GpEn versus placebo were reported in sleep, mood, and patient global assessment $(P<0.05)$. Backonja et al also compared the pharmacokinetics of GpEn and GpIR in the same patients and reported a 31\% lower daily dose of GpEn provided a significant increase in average steady-state plasma Gp concentration versus GpIR.

\section{Safety and tolerability issues}

The most frequently reported side effects of Gp are dizziness and somnolence. ${ }^{22}$ Weight gain and headache are also reported, with these adverse events being reported in both GpIR and GpGr preparations. ${ }^{11}$ Not enough data is available to ascertain whether GpEn will have a different profile from that of the immediate release form. In the absence of such information, and considering the claim that GpEn is purely a prodrug of Gp, the profile of GpIR should be strongly considered alongside any knowledge of the issues relating to GpEn. Side effect data for GpEn are summarized in Table 1.

A pharmacokinetics and pharmacodynamics study amalgamating 12 Phase I-III studies of GpEn treatment examined data collected after 2 weeks of receiving GpEn. The study mathematically predicted the probabilities that dizziness and somnolence would be lower than $5 \%$ for groups receiving 
doses of $600 \mathrm{mg}$ and 1,200 mg. ${ }^{16}$ No appreciable differences were subsequently observed between these two dosing groups for dizziness or somnolence in this data set. Subjects receiving higher doses of 1,800 $\mathrm{mg}$ and 2,400 $\mathrm{mg}$ had significantly higher frequencies of these adverse events than both the lower dose groups and the placebo-treated groups. In the paper by Zhang et al, GpEn was noted to cause more somnolence than placebo at doses above $1,200 \mathrm{mg} /$ day. $^{20}$ Somnolence was reported in this study in $10 \%$ of the patients treated with $1,200 \mathrm{mg}, 11 \%$ of those treated with $2,400 \mathrm{mg}$, and $14 \%$ of the $3,600 \mathrm{mg}$ treated group compared to $8 \%$ of the placebo group.

The age range of sufferers of PHN can span decades, but the majority of sufferers are aged over 50 years, with incidence doubling by the age of $80 .^{3}$ The two primary papers investigating GpEn included patients aged up to 82 and 92 years, respectively, with Zhang et al stating at least $9 \%$ of all arms had patients aged $>75$ years. ${ }^{20,21}$ No specific data were described investigating GpEn in the older population. It is notable that this age group might be the primary recipients of extended release $\mathrm{Gp}$ for this indication. Data on antineuropathic analgesic agents in the older population for GpIR raise concern regarding the same issue of relative age and pharmacodynamics. ${ }^{23}$ Bowel absorption is altered in the elderly, multiple medications are coprescribed, and underlying physiological systems are increasingly fragile. Where comparative data exists for immediate release preparations, GpIR is generally considered safer than TCA; however, where administered as GpEn, no specific data exists. ${ }^{23}$

With regards to weight gain, Zhang et al reported weight increase simultaneously across the three treatment groups as well as in the placebo group..$^{20}$ The increase was noted to be dose-related. Weight was then reduced in the taper period, although it persisted in the placebo group. Dizziness and nausea were reported at higher rates than placebo in a further tolerability study, with somnolence additionally appearing, amongst other symptoms, at doses greater than 3,600 $\mathrm{mg}$, which is above the licensed dose for GpIR in the United Kingdom. ${ }^{24}$ Other data taken from controlled trials in patients diagnosed with RLS reported that $20 \%$ of patients suffered somnolence or sedation at $600 \mathrm{mg}$ compared to $6 \%$ in patients taking placebo, with symptoms persisting past treatment in $30 \% .{ }^{17}$ Dizziness at the same doses was seen in $13 \%$ compared to $4 \%$ for placebo.

Since US FDA approval, a randomized, double-blind trial of placebo and active-controlled crossover design has investigated the effect of GpEn in 54 adults for cardiac repolarization $(\mathrm{QT} / \mathrm{QTc})$ changes. Therapeutic dosing of
GpEn (1,200 mg) and supramaximal dosing $(6,000 \mathrm{mg})$ produced no evidence of QT prolongation. ${ }^{25}$ Where other studies investigating the pharmacokinetics and tolerability of GpEn have investigated QT/QTc changes (with a secondary aim of being a pilot in anticipation of a definitive study), no significant effects over dosing ranges of $2,400-6,000 \mathrm{mg}$ were found..$^{24}$

The US FDA prescribing information lists suicide risk in the adverse events based on the existing evidence pertaining to Gp; pooled data show an approximate doubling of the risk with $\mathrm{Gp}$ versus placebo to $0.43 \%{ }^{17}$

No deaths or absolute contraindications to GpEn have been reported. Noninterchangeability with other preparations of $\mathrm{Gp}$ is stressed, with the extended and immediate release being clearly distinct. ${ }^{17}$ Interaction studies of GpEn have also been conducted with naproxen and cimetidine. ${ }^{26}$ One study placed 12 patients into three arms. Patients were dosed with 1,200 mg of GpEn until steady-state was achieved, and subsequently given either naproxen (500 $\mathrm{mg}$ bd), or cimetidine (400 $\mathrm{mg}$ four times daily), followed by the combination. There was no reciprocal increase in naproxen or cimetidine exposure. The study reported that there was no requirement for dosing adjustment with either of these two medications.

Other notable issues associated with GpEn have been raised in the literature such as possible impairment to driving. GpEn has also been associated with a rare drug reaction with eosinophilia and systemic symptoms, ${ }^{17}$ and has been reported in patients taking antiepileptics (including Gp). Some of these reactions have been life threatening or fatal. Advice calls for immediate clinical evaluation of the patient if signs or symptoms appear. A single oral carcinogenicity study of GpEn in rats showed an increase in the incidence of pancreatic acinar cell adenoma, but US FDA literature concludes the clinical significance of this is unknown.

\section{Place in therapy}

Therapeutic intervention for PHN treatment has no global consensus guidelines but can be managed under the guidelines available for neuropathic pain treatment. ${ }^{7-10} \mathrm{Gp}$ is included in most published national and international guidelines, either as sole agent or in combination with other antineuropathic medications. GpEn demonstrates novel technology which provides dose proportional, extended exposure to Gp that is less variable with increasing dose compared to immediate release preparations. However, no comparative data exists between the different preparations, and from an efficacy perspective, no evidence base exists to favor the choice of 
GpEn over other preparations. Additionally, the lack of data in the older population of PHN sufferers leaves the potentially highest age group who might receive GpEn for PHN without specific investigation.

While a deciding factor for some clinicians and patients may be the recommended bd dosing regimen, no efficacy benefit has been confirmed. Other preparations are available that can provide the same bd dosing, which is associated with better patient compliance and acceptance. ${ }^{11}$ It is notable that pregabalin can also be dosed in a bd regimen. ${ }^{27}$ The position of GpEn within treatment options would be best evaluated by further investigation that would inform clinicians of comparative data, and subsequently aim to rank first line oral antineuropathic agents in order to facilitate a choice based on efficacy. These gaps in the data expose our lack of comparative evidence for many antineuropathic agents in many types of neuropathic pain, including PHN.

Fundamentally, the concept of this prodrug is afforded by novel technology, to provide a vehicle that is pharmacologically altered to ensure a robust, stable, and predictable presentation of a medication subsequently metabolized to active Gp. The presumption that the variable absorption profile adversely affects the efficacy of GpIR has never been proven; therefore it is difficult to invoke a clear and tangible clinical advantage for the more predictable absorption of GpEn. Nevertheless, compared to other Gp products, GpEn appears to have equivalent efficacy with the potential advantages of a bd dosing schedule and conceivably, a lower dose.

\section{Conclusion}

GpEn is a novel preparation exhibiting tailored pharmacological engineering to overcome the pharmacokinetic limitations of Gp that are perceived to limit efficacy (although unproven). US FDA licensing has been granted based on evidence pertaining to comparison against placebo but not against other preparations. However, this criticism can be leveled at many antineuropathic pain medications. Consequently, although GpEn has some evidence to support its use (and has been licensed for this indication by the US FDA) the lack of comparative data makes it difficult to identify clearly its position in the treatment of PHN.

\section{Disclosure}

PFS has received remuneration from Pfizer for educational services. The other author reports no conflicts of interest in this work.

\section{References}

1. Doggrell SA. Pregabalin or morphine and gabapentin for neuropathic pain. Expert Opin Pharmacother. 2005;6(14):2535-2539.

2. Gupta R, Farquhar-Smith P. Post-herpetic neuralgia. Contin Educ Anaesth Crit Care Pain. 2012;12(4):181-185.

3. Bader MS, McKinsey DS. Viral infections in the elderly. The challenges of managing herpes zoster, influenza, and RSV. Postgrad Med. 2005;118(5):45-48, 51-54.

4. Wareham DW, Breuer J. Herpes zoster. BMJ. 2007;334(7605): 1211-1215.

5. Edmunds WJ, Brisson M, Rose JD. The epidemiology of herpes zoster and potential cost-effectiveness of vaccination in England and Wales. Vaccine. 2001;19(23-24):3076-3090.

6. Richter A, Anton SE, Koch P, Dennett SL. The impact of reducing dose frequency on health outcomes. Clin Ther. 2003;25(8):2307-2335.

7. Dworkin RH, O'Connor AB, Backonja M, et al. Pharmacologic management of neuropathic pain: evidence-based recommendations. Pain. 2007;132(3):237-251.

8. Dworkin RH, O'Connor AB, Audette J, et al. Recommendations for the pharmacological management of neuropathic pain: an overview and literature update. Mayo Clin Proc. 2010;85(Suppl 3):S3-S14.

9. American Society of Anesthesiologists Task Force on Chronic Pain Management; American Society of Regional Anesthesia and Pain Medicine. Practice guidelines for chronic pain management: an updated report by the American Society of Anesthesiologists Task Force on Chronic Pain Management and the American Society of Regional Anesthesia and Pain Medicine. Anesthesiology. 2010;112(4):810-833.

10. Attal N, Cruccu G, Baron R, et al; European Federation of Neurological Societies. EFNS guidelines for the pharmacological treatment of neuropathic pain: 2010 revision. Eur J Neurol. 2010;17(9): $1113-1123$.

11. Thomas B, Farquhar-Smith P. Extended-release gabapentin in post-herpetic neuralgia. Expert Opin Pharmacother. 2011;12(16): 2565-2571.

12. Eroglu C, Allen NJ, Susman MW, et al. Gabapentin receptor alpha2delta-1 is a neuronal thrombospondin receptor responsible for excitatory CNS synaptogenesis. Cell. 2009;139(2):380-392.

13. Gupta SK, Mahajan A, Tandon V. Gabapentin for the treatment of neuropathic pain. JK Science. 2004;6(3):113-114.

14. Cundy KC, Branch R, Chernov-Rogan T, et al. XP13512 [(+/-)-1-([(alphaisobutanoyloxyethoxy)carbonyl] aminomethyl)-1-cyclohexane acetic acid], a novel gabapentin prodrug: I. Design, synthesis, enzymatic conversion to gabapentin, and transport by intestinal solute transporters. J Pharmacol Exp Ther. 2004;311(1):315-323.

15. Neurontin ${ }^{\circledR}$ (gabapentin) Caps,Tab,OS [prescribing information]. New York, NY: Pfizer Inc; 2013. Available from: http://labeling.pfizer. com/ShowLabeling. aspx?id=630. Accessed May 20, 2013.

16. Lal R, Sukbuntherng J, Luo W, Tovera J, Lassauzet ML, Cundy KC. Population pharmacokinetics and pharmacodynamics of gabapentin after administration of gabapentin enacarbil. J Clin Pharmacol. 2013;53(1):29-40.

17. US Food and Drug Administration. Highlights of prescribing information: HORIZANT ${ }^{\mathrm{TM}}$ (gabapentin encarbil). Silver Spring, MD; US Food and Drug Administration; 2011. Available from: http://www. accessdata.fda.gov/drugsatfda_docs/label/2011/022399s0001bl.pdf. Accessed May 19, 2013.

18. US Government Department of Veterans Affairs. Gabapentin Enacarbil $\left(\right.$ Horizant $\left.^{\mathbb{E}}\right)$ National Drug Monograph. Washington, DC: US Government Department of Veterans Affairs; 2012. Available from: http://www.pbm.va.gov/PBM/clinicalguidance/drugmonographs/ GabapentinEnacarbilHorizantDrugMonograph.pdf. Accessed May 21, 2013.

19. Cundy KC, Annamalai T, Bu L, et al. XP13512 [(+/-)-1-([(alphaisobutanoyloxyethoxy)carbonyl] aminomethyl)-1-cyclohexane acetic acid], a novel gabapentin prodrug: II. Improved oral bioavailability, dose proportionality, and colonic absorption compared with gabapentin in rats and monkeys. J Pharmacol Exp Ther. 2004;311(1):324-333. 
20. Zhang L, Rainka M, Freeman R, et al. A randomized, double-blind, placebo-controlled trial to assess the efficacy and safety of gabapentin enacarbil in subjects with neuropathic pain associated with postherpetic neuralgia (PXN110748). J Pain. 2013;14(6):590-603.

21. Backonja MM, Canafax DM, Cundy KC. Efficacy of gabapentin enacarbil vs placebo in patients with postherpetic neuralgia and a pharmacokinetic comparison with oral gabapentin. Pain Med. 2011;12(7): 1098-1108.

22. Moore RA, Wiffen PJ, Derry S, McQuay HJ. Gabapentin for chronic neuropathic pain and fibromyalgia in adults. Cochrane Database Syst Rev. 2011;(3):CD007938.

23. Haslam C, Nurmikko T. Pharmacological treatment of neuropathic pain in older persons. Clin Interv Aging. 2008;3(1):111-120.

24. Lal R, Sukbuntherng J, Luo W, et al. Pharmacokinetics and tolerability of single escalating doses of gabapentin enacarbil: a randomizedsequence, double-blind, placebo-controlled crossover study in healthy volunteers. Clin Ther. 2009;31(8):1776-1786.
25. Chen D, Lal R, Zomorodi K, et al. Evaluation of gabapentin enacarbil on cardiac repolarization: a randomized, double-blind, placebo- and active-controlled, crossover thorough QT/QTc study in healthy adults. Clin Ther. 2012;34(2):351-362. e3.

26. Lal R, Sukbuntherng J, Luo W, et al. Clinical pharmacokinetic drug interaction studies of gabapentin enacarbil, a novel transported prodrug of gabapentin, with naproxen and cimetidine. Br J Clin Pharmacol. 2010;69(5):498-507.

27. US Food and Drug Administration. Highlights of prescribing information: LYRICA ${ }^{\circledR}$ (pregabalin). Silver Spring, MD; US Food and Drug Administration; 2011. Available from: http://www.accessdata.fda.gov/ drugsatfda_docs/label/2011/021446s026,022488s005lbl.pdf. Accessed May 19, 2013

\section{Publish your work in this journal}

Therapeutics and Clinical Risk Management is an international, peerreviewed journal of clinical therapeutics and risk management, focusing on concise rapid reporting of clinical studies in all therapeutic areas, outcomes, safety, and programs for the effective, safe, and sustained use of medicines. This journal is indexed on PubMed Central, CAS,

\section{Dovepress}

EMBase, Scopus and the Elsevier Bibliographic databases. The manuscript management system is completely online and includes a very quick and fair peer-review system, which is all easy to use. Visit http://www.dovepress.com/testimonials.php to read real quotes from published authors.

Submit your manuscript here: http://www.dovepress.com/therapeutics-and-clinical-risk-management-journal 